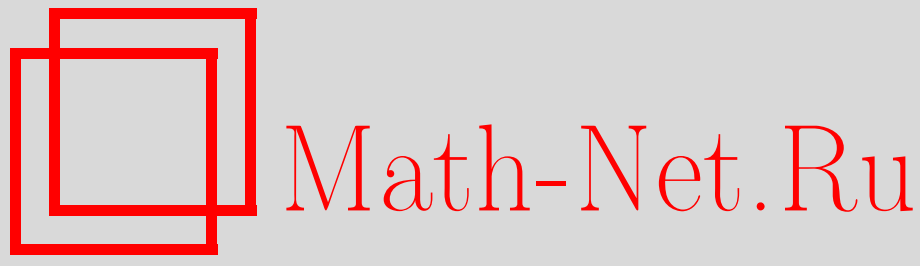

Общероссийский математический портал

И. К. Мацак, Об относительной устойчивости экстремальных случайных функций, Матем. заметки, 2002, том 71, выпуск 5, 787-790

DOI: https://doi.org/10.4213/mzm652

Использование Общероссийского математического портала Math-Net.Ru подра- 
зумевает, что вы прочитали и согласны с пользовательским соглашением http://www . mathnet.ru/rus/agreement

Параметры загрузки:

IP : 54.224 .135 .184

26 апреля 2023 г., 16:17:43

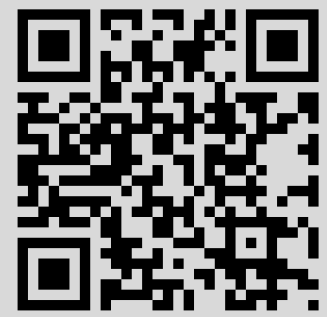




\section{ОБ ОТНОСИТЕЛЬНОЙ УСТОЙЧИВОСТИ ЭКСТРЕМАЛЬНЫХ СЛУЧАЙНЫХ ФУНКЦИЙ}

\section{И.К. Мацак}

Рассмотрим нормально распределенную случайную функцию (с.ф.) $X=\{X(t), t \in T\}$, заданную на некотором параметрическом множестве $T$ и принимающую значения в $\mathbb{R}$. Через $\sigma_{T} X$ будем обозначать среднее квадратическое отклонение с.ф. $X$ :

$$
\sigma_{T} X=\{\sigma(t), t \in T\}, \quad \sigma(t)=\left(M|X(t)-M X(t)|^{2}\right)^{1 / 2} .
$$

Для последовательности $\left(X_{n}\right)$ независимых копий $X$ введем экстремальную с.ф.

$$
Z_{n}=\left\{Z_{n}(t), t \in T\right\}, \quad Z_{n}(t)=\sup _{1 \leqslant k \leqslant n} X_{k}(t) .
$$

Будем говорить, что последовательность $\left(Z_{n}\right)$ относительно устойчива почти наверное (п.н.), если выполняется соотношение

где

$$
\lim _{n \rightarrow \infty}\left|\frac{Z_{n}}{b_{n}}-\sigma_{T} X\right|=0 \quad \text { п.н., }
$$

$$
b_{n}= \begin{cases}\sqrt{2 \ln n}, & n>1, \\ 1, & n=1,\end{cases}
$$

$\|x\|=\sup _{t \in T}|x(t)|-$ равномерная норма.

Если для вещественной прямой относительная устойчивость экстремальных значений последовательности случайных величин (с.в.) и ее обобщения широко изучались (см. [1]-[3]), то бесконечномерньй случай исследовался значительно меньше. Относительная устойчивость экстремальных случайных элементов (с.э.) установлена для пространства $C[0,1]$ и для банаховых решеток, не содержащих равномерно $l_{\infty}^{n}$ (см. [4]).

В данной заметке доказывается справедливость равенства (1) для произвольной ограниченной нормальной с.ф., т.е. $\|X\|<\infty$ п.н. 
Теорема. Пусть $X$ - ограниченная нормально распределенная с.ф. Тогда последовательность әкстремальных с.ф. $\left(Z_{n}\right)$ относительно устойчива п.н.

При доказательстве теоремы будут использоваться следующие вспомогательные утверждения.

Лемма 1 [5]. Пусть $X$ - ограниченная нормально распределенная с.ф. Тогда существует нормальная последовательность с.в. $\left(\xi_{k}\right)$ такая, что $\left(M\left|\xi_{k}\right|^{2}\right)^{1 / 2} \leqslant K M\|X\| / b_{k} u$ для любого $t \in T$

$$
X(t)=\sum_{k \geqslant 1} \xi_{k} a_{k}(t)
$$

где $\sum_{k \geqslant 1} a_{k}(t) \leqslant 1, a_{k}(t) \geqslant 0, K-$ абсолютная константа, а ряд сходится п.н. и в $L_{2}$.

Мы приводим несколко более грубую оценку для величины $\left(M\left|\xi_{k}\right|^{2}\right)^{1 / 2}$, чем в работе [5].

ЛЕмма 2. Если $X$-ограниченная нормально распределенная с.ф., то

$$
\lim _{n \rightarrow \infty}\left\|\frac{Z_{n}}{b_{n}}\right\|=\left\|\sigma_{T} X\right\|
$$

Лемма 2 следует из результатов работы [6].

ДоКАЗАТЕЛЬСТво ТЕОРЕмы. Воспользуемся представлением (2) для с.ф. $X$. Обозначим через $X_{n}^{(m)}(t)$ независимые копии $X^{(m)}(t)$,

$$
X^{(m)}(t)=\sum_{1 \leqslant k \leqslant m} \xi_{k} a_{k}(t), \quad Z_{n}^{(m)}(t)=\sup _{1 \leqslant k \leqslant n} X_{n}^{(m)}(t), \quad n \geqslant 1 .
$$

По неравенству треугольника

$$
\left\|\frac{Z_{n}}{b_{n}}-\sigma_{T} X\right\| \leqslant\left\|Z_{n}-Z_{n}^{(m)}\right\| b_{n}^{-1}+\left\|\frac{Z_{n}^{(m)}}{b_{n}}-\sigma_{T} X^{(m)}\right\|+\left\|\sigma_{T} X^{(m)}-\sigma_{T} X\right\| .
$$

Поскольку с.э. $X^{(m)}$ лежит в конечномерном пространстве, а для таких пространств равенство (1) выполняется (это следует из вложения конечномерного пространства в пространство $\bar{H}_{m}$ всех непрерывных функций из $\mathbb{R}^{m}$ в $\mathbb{R}$, однородных степени $1[7$, с. 42$\left.]\right)$, то п.н.

$$
\lim _{n \rightarrow \infty}\left\|\frac{Z_{n}^{(m)}}{b_{n}}-\sigma_{T} X^{(m)}\right\|=0 .
$$

Без ограничения общности можно считать, что $M X(t)=0$. Тогда

$$
\sigma(t)=\left(M|X(t)|^{2}\right)^{1 / 2}=\sqrt{\frac{\pi}{2}} M|X(t)| .
$$

Отсюда и из леммы 1 получаем

$$
\begin{aligned}
\left\|\sigma_{T} X^{(m)}-\sigma_{T} X\right\| & =\sqrt{\frac{\pi}{2}} \sup _{t \in T}|M| X^{(m)}(t)|-M| X(t)|| \leqslant \sqrt{\frac{\pi}{2}} \sup _{t \in T} M\left|\sum_{k \geqslant m} \xi_{k} a_{k}(t)\right| \\
& \leqslant \sqrt{\frac{\pi}{2}} \sup _{t \in T} M \sum_{k \geqslant m}\left|\xi_{k}\right| a_{k}(t) \leqslant \sqrt{\frac{\pi}{2}} K M \frac{\|X\|}{b_{m}} .
\end{aligned}
$$

Далее оценим сверху первое слагаемое в правой части неравенства (4). При этом восползуемся леммой 2 и элементарньм неравенством

$$
\left|\max _{1 \leqslant k \leqslant n} c_{k}-\max _{1 \leqslant k \leqslant n} d_{k}\right| \leqslant \max _{1 \leqslant k \leqslant n}\left|c_{k}-d_{k}\right| .
$$


Имеем

$$
\begin{aligned}
\limsup _{n \rightarrow \infty}\left\|Z_{n}-Z_{n}^{(m)}\right\| b_{n}^{-1} & \leqslant \limsup _{n \rightarrow \infty}\left\|\sup _{1 \leqslant k \leqslant n} X_{k}(t)-\sup _{1 \leqslant k \leqslant n} X_{k}^{(m)}(t)\right\| b_{n}^{-1} \\
& \leqslant \limsup _{n \rightarrow \infty}\left\|\sup _{1 \leqslant k \leqslant n}\left|X_{k}(t)-X_{k}^{(m)}(t)\right|\right\| b_{n}^{-1} \leqslant\left\|\sigma_{T}\left(X^{(m)}-X\right)\right\| .
\end{aligned}
$$

Из цепочки неравенств (6) нетрудно вывести оценку

$$
\left\|\sigma_{T}\left(X^{(m)}-X\right)\right\| \leqslant \sqrt{\frac{\pi}{2}} K M \frac{\|X\|}{b_{m}} .
$$

Для завершения доказательства теоремы остается заметить, что соотношения (4)-(8) содержат следующее неравенство:

$$
\limsup _{n \rightarrow \infty}\left\|\frac{Z_{n}}{b_{n}}-\sigma_{T} X\right\| \leqslant \sqrt{2 \pi} K M \frac{\|X\|}{b_{m}} .
$$

Действительно, поскольку $m$ - произвольное число, а $b_{m} \rightarrow \infty$ при $m \rightarrow \infty$, отсюда немедленно получаем равенство (1).

Для банаховой решетки $E$ обозначим через $E_{(u)}$ идеал, порожденный положительным элементом $u$, т.е. $E_{u}=\{x \in E: \exists \lambda>0,|x| \leqslant \lambda u\}$. Тогда $E_{(u)}$ с нормой $\|x\|_{u}=\inf \{\lambda>0:|x| \leqslant \lambda u\}$ будет банаховой решеткой, $(o)$-изометричной пространству $C(K)$ для некоторого компактного пространства Хаусдорфа $K$ [8]. Известно, что (o)-изометрия банаховых решеток сохраняет порядок, модуль, верхнюю и нижнюю грани. Следовательно, из теоремы имеем

СледствиЕ. Пусть $X-$ нормальный случайный әлемент со значениями в сепарабельной банаховой решетке $E,\left(X_{n}\right)$ - последовательность независимых копий $X$,

$$
Z_{n}=\sup _{1 \leqslant k \leqslant n} X_{k}, \quad \sigma_{T} X=\sqrt{\frac{\pi}{2}} M|X-M X| .
$$

Если существует полохительный элемент и такой, что $\|X\|_{u}<\infty$ n.н., то последовательность $Z_{n}$ будет относительно устойчива п.н.; более того, существует последовательность с.в. $\left(\tau_{n}\right)$ такал, что $\tau_{n} \mapsto 0$ п.н. при $n \rightarrow \infty u$

$$
\left|\frac{Z_{n}}{b_{n}}-\sigma_{T} X\right| \leqslant \tau_{n} u \quad \text { n.н. }
$$

ПримеР. В одномерном случае известно равенство

$$
\lim _{n \rightarrow \infty}\left\|Z_{n}-b_{n} \sigma_{T} X\right\|=0 .
$$

Возникает вопрос: нельзя ли в условиях теоремы усилить относительную устойчивость до равенства (9)?

Ответ отрицательный. В работе [9] построен пример нормального случайного процесса $X$ такого, что $\left\|\sigma_{T} X\right\|=1,\|X\|<\infty$ п.н. и

$$
M \exp \left(\frac{\|X\|^{2}}{2}-(1+\varepsilon)\|X\|\right)=\left\{\begin{array}{cc}
+\infty & \text { при } \varepsilon=0 \\
<\infty & \text { при } \varepsilon>0 .
\end{array}\right.
$$

Известно [10], что для ограниченной нормальной с.ф. $X$ существует число $d$ такое, что

$$
\lim _{r \rightarrow \infty} r^{-1}\left\{\log P(\|X\| \geqslant r)+\frac{(r+d)^{2}}{2\left\|\sigma_{T} X\right\|^{2}}\right\}=0 .
$$

Из двух последних равенств имеем $d=-1$. Тогда (см. [6]) для такого процесса выполняется равенство

что противоречит (9).

$$
\lim _{n \rightarrow \infty}\left\|Z_{n}\right\|-b_{n}\left\|\sigma_{T} X\right\|=1 \quad \text { п.н. }
$$

Таким образом, для любого пространства $C(K)$ выполняется равенство (1). С другой стороны, существует компактное пространство Хаусдорфа $K$ и нормальный с.э. $X$ со значениями в пространстве $C(K)$, для которого равенство (9) не выполняется. 


\section{СПИСОК ЦИТИРОВАННОЙ ЛИТЕРАТУРЫ}

1. Gnedenko B. V. // Ann. Math. 1943. V. 44. P. 423-453. 2. Barndorf-Nielsen O. // Ann. Math. Statist. 1963. V. 34. № 3. P. 992-1002. 3. Галамбош Я. Асимптотическая теория экстремальньх порядковых статистик. М.: Наука, 1984. 4. Мацак I. К. // Укр. матем. ж. 1995. T. 47. № 7. C. 1006-1008. 5. Ledoux M., Talagrand M. Probability in Banach Spaces. Berlin: Springer, 1991. 6. Мацак I. К. // Теорія ймовірностей та матем. статист. 1997. № 56. С. $126-132$. 7. Lindenstrauss J., Tzafriri L. Classical Banach Spaces. V. 2. Berlin: Springer, 1979. 8. Kaнторович Л. В., Акилов Г. П. Функциональный анализ. М.: Наука, 1984. 9. Talagrand M. // Zeitschrift für Warhscheinlichkeitstheor. verw. Geb. 1984. V. 68. № 1. P. 1-8. 10. Лифшиц М. А. Гауссовские случайные функции. Киев: ТвіМС, 1995.

г. Киев 